\title{
Remarkable Advances in the Management of Mycosis Fungoides and the Sezary Syndrome
}

\author{
Richard T. Hoppe \\ Department of Radiation Oncology - Radiation Therapy, Stanford University, Stanford, CA, USA
}

In 1978, a Workshop on Cutaneous T-Cell Lymphomas was held at the National Cancer Institute (NCI), Bethesda, Maryland. At the conclusion of that workshop, a consensus statement was developed regarding therapy for mycosis fungoides and the Sezary syndrome [1]. Based on a review of knowledge at the time, this consensus statement focused on the following treatments: highdose total skin electron beam therapy (TSEBT), topical mechlorethamine, methoxsalen photochemotherapy (PUVA), and systemic chemotherapy. The excellent response rates to TSEBT were noted, but the challenge of maintaining that response was acknowledged and early trials of combined modality therapy with topical or systemic agents were cited. The disappointing results of treatment with conventional chemotherapy were tabulated. The potential utility of biologic therapies was barely noted, only anti-thymocyte globulin was mentioned, with the point being made that novel agents required documentation of response in phase II clinical trials.

We have come a long way since 1978! Our understanding of the disease, the importance of the skin microenvironment, the discovery and development of new biologic therapies, and the refinement of conventional therapies has led to our ability to manage the disease more effectively, improve the quality of life of our patients, and potentially extend their life expectancy, as well.

In their paper on the use of innovative concepts treating the microenvironment in cutaneous T-cell lymphoma, Leuchte et al. [2] describe more than 20 agents that have been tested in clinical trials or are in current clinical use. The treatment guidelines from the National Cancer Center Network (NCCN) [3], which are accessed world-wide, include 2 topical and 10 systemic biologics as already acceptable for use in clinical practice. Although the response rates and duration of response to these therapies are quite variable, there is little doubt that these agents have contributed significantly to improving the quality of life for our patients. In addition, the use of more targeted therapies, such as brentuximab vedotin, provides an opportunity to treat the disease with fewer side effects than would be expected with systemic chemotherapy.
Given these and the many other agents in the 'pipeline', patients in the future will have many options for keeping their disease in control.

The refinement of existing therapies is another factor that has led to improvement in outcomes. Both Elsayad et al. [4] and Dabaja et al. [5] in their papers discuss the reconsideration of radiation therapy dose for managing mycosis fungoides. In 1978, at the time of the NCI workshop, with so few therapies being available for treatment, the philosophy was to maximize management with any single modality in order to optimize outcome. Complete response rates achieved with TSEBT improved with increasing dose, and were greatest with 36 Gy [6]. Although this is true, the overall response rates are high, even with low dose ( 12 Gy) TSEBT [7]. Since the primary role of TSEBT is to minimize the disease burden, we now realize that this can be achieved with those lower doses, with intent then to keep patients in a minimal disease state using the multitude of other therapies now available. The advantages of this approach include patient convenience, reduction of side effects, and the opportunity to utilize TSEBT more often in the course of a patient's disease, which may extend over many years. This approach has been tested in prospective trials, where the duration of clinical benefit (time to requiring another total skin or systemic therapy) from a single course of 12 Gy TSEBT was 70 weeks [8].

The ultimate utility of TSEBT would be realized if an effective adjuvant therapy could be identified that would reliably maintain the response achieved. Elsayad et al. [4] provide a table summarizing the use of adjuvant therapies in this setting. Most of the experience relates to mechlorethamine, PUVA and miscellaneous therapies. All of the data are retrospective and non-randomized. Although some of these reports claim a prolongation of response with adjuvant therapy, none are associated with permanent disease control. What is clearly desirable are prospective clinical trials, preferably randomized, that test the value of adjuvant biologic therapy following TSEBT. For example, TSEBT followed by randomization to brentuximab vedotin versus no further therapy.

\section{KARGER}

() 2017 S. Karger GmbH, Freiburg
Richard T. Hoppe, M.D.

Department of Radiation Oncology - Radiation Therapy

Stanford University

875 Blake Wilbur Drive, Room CC-G228, Stanford CA 94305, USA

rhoppe@stanford.edu 
The Sezary syndrome variant of mycosis fungoides has been associated with very poor outcomes. In addition, it is often the most symptomatic, with unremitting pruritus and scaling. Interestingly, this variant of the disease may be the most responsive to biologic therapy. In addition, there is now mounting evidence that allotransplantation, which allows for an immune-mediated graft versus lymphoma effect, may be curative in a subpopulation of these patients [2]. The most effective conditioning regimens have not been identified, and the reason for failure remains recurrence of disease, but this general approach holds important promise for the management of these patients.
Further advances in the treatment of mycosis fungoides and the Sezary syndrome are inevitable. Although it is an 'orphan' disease, it has attracted numerous talented dermatologists, pathologists, hematologists and oncologists, who are studying it with a passion. It is not unrealistic to expect the development of even more effective management programs in the next decade.

\section{Disclosure Statement}

The author has no conflicts to disclose.

\section{References}

1 Minna JD, Roenigk HH, Glatstein E: Report of the Committee on Therapy for Mycosis fungoides and Sezary Syndrome. Cancer Treat Rep 1979;63:729-736.

2 Leuchte K, Schlaak M, Stadler R, Theurich S, von Bergwelt-Baildon M: Use of innovative concepts treating the microenvironment in cutaneous T-cell lymphoma. Oncol Res Treat 2017;40:DOI: 10.1159/000472257.

3 NCCN Clinical Practice Guidelines in Oncology: T-cell lymphomas. https://www.nccn.org/professionals/ physician_gls/pdf/t-cell.pdf (accessed 31 March 2017).
4 Elsayad K, Susek K, Eich HT: Total-skin electron beam treatment as part of multimodal therapeutic strategies for the treatment of primary cutaneous T-cell lymphoma. Oncol Res Treat 2017;40:DOI: $10.1159 / 000475634$

5 Dabaja B: Renaissance of low-dose radiotherapy concepts for cutaneous lymphomas. Oncol Res Treat 2017; 40:DOI: 10.1159/000470845.

6 Hoppe RT, Fuks Z, Bagshaw MA: Radiation therapy in the management of cutaneous T-cell lymphomas. Cancer Treat Rep 1979;63:625-632.
7 Harrison C, Young J, Navi D, Riaz N, Lingala B, Kim Y, Hoppe R: Revisiting low-dose total skin electron beam therapy in mycosis fungoides. Int J Radiat Oncol Biol Phys 2011;81:e651-7.

8 Hoppe RT, Harrison C, Tavallaee M, Bashey S, Sundram U, Li S, Million L, Dabaja B, Gangar P, Duvic M, Kim YH: Low-dose total skin electron beam therapy as an effective modality to reduce disease burden in patients with mycosis fungoides: results of a pooled analysis from 3 phase-II clinical trials. J Am Acad Dermatol 2015;72:286-92. 